\title{
A PIV-based method to measure spatial gradients in bedload transport over a dune
}

\author{
Renske C. Terwisscha van Scheltinga ${ }^{1, *}$, Heide Friedrich ${ }^{1}$, and Giovanni Coco $^{2}$ \\ ${ }^{1}$ University of Auckland, Faculty of Engineering, University of Auckland, New Zealand \\ ${ }^{2}$ Auckland, Faculty of Science, Auckland, New Zealand
}

\begin{abstract}
Traditional sediment transport equations calculate sediment flux from bed shear stress and the equations predict that transport increases nonlinearly with an increase in flow velocity. In a dune field, the dune geometry affects the flow velocity causing accelerating flow over the dune crest and de- and reattachment of the flow downstream of the dune crest. Sediment flux predicted from the reach-averaged bed shear stress gives fairly good results for dune fields, though their simplification is discordant for the complexity of the processes involved. Measurements of the displacement of sand particles over the dune bed were derived from highfrequency image capturing. The two main methods to measure particle velocities from images are particle tracking velocimetry (PTV) and particle image velocimetry (PIV). We compare individual particle tracking with a PIV-based correlation method. The PIV-based method promises to be a more efficient and effective approach to track particle motion. It is more suitable for the conditions of high bedload transport, as present in our experiments. The PIV-based method is based on using images of difference (IoD) and is fully automated and identifies spatial gradients at a support scale in the order of centimetres. Findings align with our general knowledge of accelerating flow over the dune crest. The mean streamwise particle velocity and activity over a dune stoss slope increase. At the scale of $0.026 \mathrm{~m}$ the observed particle velocity variability can be explained in the context of general onset and cessation of sediment transport, the effect of the reattachment zone and observed sweep/burst events. By decreasing the streamwise distance between cross-sections, the variations in mean particle velocity induced by superimposed bed defects are distinguished as well. The maximum particle velocity and activity occurred at the same location and consequently the location of the maximum transport over the dune crest was identified. The measurements bridge the gap between individual particle motion studies and (non-local) sediment transport flux measurements.
\end{abstract}

\section{Introduction}

Dunes are formed at the boundary between the loose sediment and the flow. The form of the dune causes the flow to separate in the lee of the dune, and reattach 4-6 dune heights

* Corresponding author: rgal477@,aucklanduni.ac.nz 
downstream of the crest [1]. Downstream of the flow reattachment zone, the boundary shear layer grows and redevelops [2]. At the same time, the form of the dune effectuates accelerating flow due to flow conversion caused by the decreasing water depth over the crest. The maximum horizontal velocity therefore occurs over the dune crest [1]. The location of maximum transport is crucial for understanding dune growth [3,4], although it remains challenging to model the complex interaction between flow resistance and sediment transport. Consequently, experimental and field data remain key input data for studies on dune kinematics [5].

A variety of studies have shown that image analysis can provide valid measurements of sediment transport at the bed [6-11], though it has so far not been applied widely in the study of dunes. The two main methods to measure particle velocities from images are particle tracking velocimetry (PTV) and particle image velocimetry (PIV) [12]. PIV is suitable for measuring velocities of multiple moving particles, while PTV is used to investigate the velocity, density and transport rate of individual particles [11]. We explored both methods to measure particle motion over a dune slope and in the present work we expand on previous studies, addressing the following research questions: (1) What are the support scales and application ranges of using mean of particle motion to quantify sediment transport over dunes compared to tracking individual particle motion? (2) What is the gradient in sand transport over the dune crest, assuming flow accelerates over the dune slope and at what location does the maximum flux occur?

\section{Particle motion tracking}

\subsection{Individual particle tracking}

A range of experimental and field studies have measured characteristics of particle motion of individual particles based on PTV or manual tracking [6, 7, 11, 13-15]. Manual tracking is the conventional method and is extremely time-consuming [13]. PTV measures the velocity and the flow path of individual particles in a fluid and can be automated when moving particles are segmented against the background by e.g. seeding with dyed particles, illumination of moving particles, or using a background with distinct colour from the particles [16]. Particle motion is intrinsically variable because the onset of a particle movement occurs randomly, while the motion can be divided into phases of acceleration and deceleration [6], such as illustrated for saltation particles in Fig. 1b. Studies of tracking particle motion for a large number of individual particles $(1,000-10,000)$ have provided distributions of the particle velocities that can be fitted by an exponential law that monotonically decreases to zero for the streamwise direction, and a Gaussian function in the cross-stream direction $[6,7,11,13]$. The average of a particle velocity is dominated by the mean value of the bed shear stress and increases linearly for the domain close to initiation of motion $[6,11]$. The relations that were retrieved using PTV and manual tracking were obtained at a limited range of conditions of flow velocity, water depth, sediment sizes and were for the situation of a flat bed, suggesting that they are not necessarily applicable to the situation of dunes with (i) variation in sediment sizes and (ii) non-uniform flow over the dune slope and (iii) higher mobility conditions. In case of higher mobility conditions, more particles move in an analysis area. Particles tend to move closer together and it becomes more challenging to automatically detect individual particle motion, and to analyse the motion. The latter is illustrated in Fig. 1c and Fig. 1d. In subsequent sections we evaluate an alternative option, based on PIV, to individual particle tracking for sediment flux measurements. 


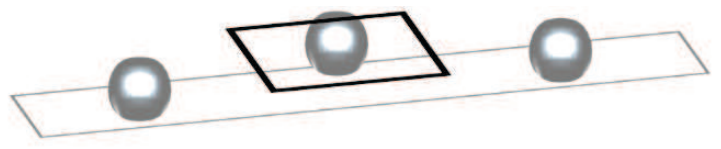

a)

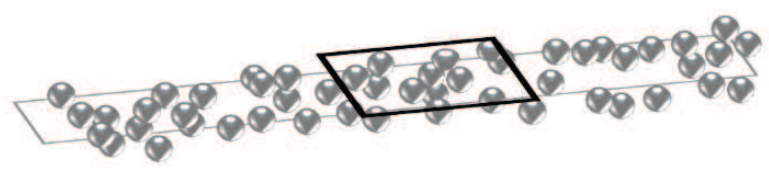

c)

Particle in motion

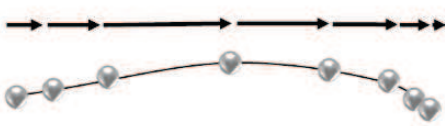

b)

d)

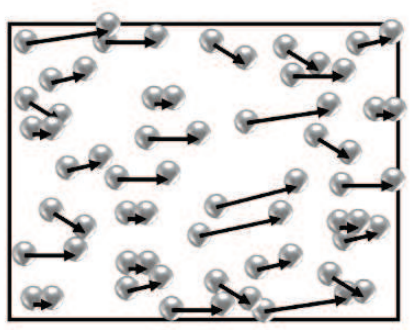

Analysis area $\longrightarrow$ Velocity Vector/Displacement per time step

Fig. 1. Particles in motion over a surface $(a, c)$. Important differences are the number of particles in motion; the proximity of particles in motion; the size of the particles, (a) allows for the calculation of individual particle motion, while in (c) individual particle tracking is challenging because particles move close together. (b) Sketch of flight trajectory of a saltation particles, showing long flights with a short phase of acceleration and a longer phase of decreasing velocity. (d) Plan view of particle motion, indicating that for uniform and steady flow conditions particles move at different velocities and in different cross-stream directions.

\subsection{PIV-based particle motion tracking with the use of images of difference}

The moving particles are filtered from the immobile particles by image subtraction, creating an image of difference (IoD). This step eliminates the need of particle segmentation by using tracer particles or by visual (manual) tracking of motion [10, 11]. Measuring the velocities of all moving particles over a moving bed from a filtering procedure that detect edges of the moving particles in IoD, Shim and Duan [11] achieved to calculate bedload transport rate with a mean error of 5\% (compared to a conventional sediment trap). In our experiments sediment size is smaller, with a median grain size of $0.85 \mathrm{~mm}$, compared to a median grain size of $1.5 \mathrm{~mm}$ and $2.4 \mathrm{~mm}$ in the experiments of Shim and Duan [11]. The Shields parameter in our experiments is higher, with more particles being in motion (compare Fig. 1a to c). To facilitate a faster calculation of the particle velocities, Radice, et al. [10] proposed to calculate the mean particle velocity using PIV. PIV measures the velocity field of a fluid at a grid, as seen from a fixed point in space. The fluid is seeded with tracer particles that are assumed to follow the flow dynamics. PIV has proven suitable for measurements of flow velocity [17-19] and provided detailed information on flow structures, such as large-scale coherent flow structures above dunes [18]. Tools for the application of PIV are readily available. An example of a tool to apply PIV is PIVlab, which bases particle displacement on the cross-correlation between groups of particles in two subsequent images [19], obtaining the most probable displacement. Radice, et al. [10] applied PIV on particles moving over the bed, using images of difference (IoD) for tracking bedload. Previously, the more conventional approach was to use seeded particles that were immersed in the flow. Radice, et al. [10] acquired a mean of the streamwise velocity of the bedload $(1.9 \mathrm{~mm})$ on a flat bed using PIV, and calculated sediment flux with a mean error of $25 \%$. We used a similar procedure as Radice, et al. [10], with IoD as input to PIVlab, to calculate mean particle velocities over a dune slope and results provided good agreement 
with measurements of particle velocities obtained by manual tracking. The particle velocity changes as a function of slope. On average, the particle velocity increases over the dune slope. Several processes occur simultaneously and explain the spatial variability in the mean particle velocity: (i) the randomness of sediment onset and cessation; (ii) the effect of flow structures; and (iii) the reattachment of the shear layer over a dune. Concluding, with the PIV-based method we are able to identify spatial gradients in streamwise velocity of particles over a dune slope. Additionally, calculation of number of moving particles or fraction of the bed in motion is needed to measure the mass flux. For the latter, the IoD, ideally, should cover (i) all the moving particles and (ii) the volume of the particles can be derived from the plan view images and (iii) the sediment transport layer has a maximum thickness of one particle size.

\section{Experimental setup and data collection}

Experiments were undertaken at the Water Engineering Laboratory (WEL) at the University of Auckland in an 11.9 meter long, $0.38 \mathrm{~m}$ deep and $0.44 \mathrm{~m}$ wide glass-sided open channel. Flow discharge was $0.033 \mathrm{~m} / \mathrm{s}$ and the flume was tilted at a slope of 0.0015 (). The initial bed level was $0.10 \mathrm{~m}$ and the average water level was $0.15 \mathrm{~m}$. Median grain diameter of the mixed sand was $0.85 \mathrm{~mm}$. Data collection was done through high-frequency image capturing at plan view. A $1.1 \mathrm{~m}$ long acrylic sheet of $4 \mathrm{~mm}$ thickness was placed on the water surface to reduce refraction and the occurrence of water surface waves $[8,20]$. Here, a selection of the data is used containing two image series, each $10 \mathrm{~s}$ of measurements at $90 \mathrm{fps}$ of moving particles over a dune crest (Fig. 2). Our measurements over the dune crest were spatially averaged for $0.026 \mathrm{~m}$ in the streamwise direction and cover a width of $0.21 \mathrm{~m}$. The lee slope was steep and showed avalanching processes. Dune height is $0.045 \mathrm{~m}$.

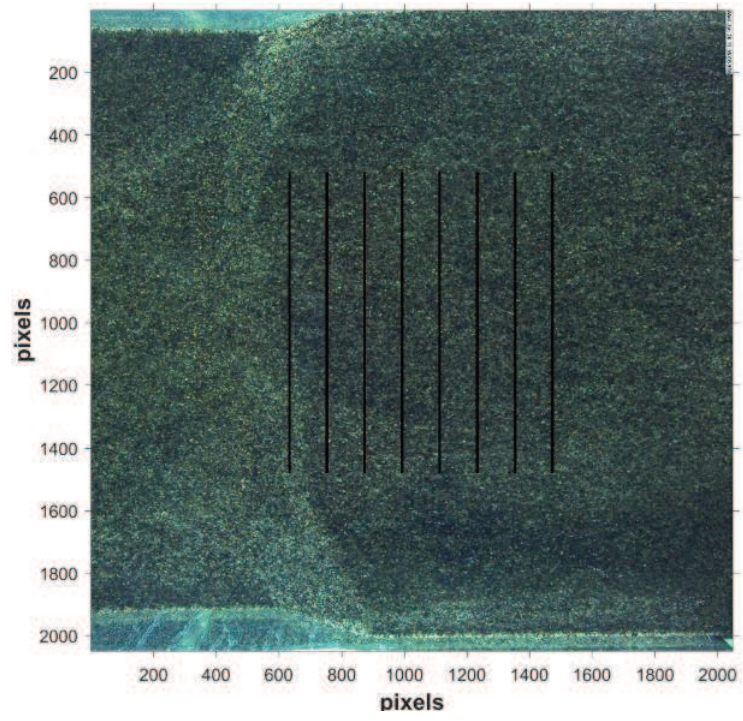

Fig. 2. Example of an image showing plan view over the dune crest and part of the upper slope. Measurements are taken at the cross-sections (black lines), which are located $0.026 \mathrm{~m}$ (120 pixels) apart. Image resolution is 2048 by 2048 . 


\section{Support scale of analysis}

PIV is sensitive to the choice of the size of the interrogation area (IA), among other factors such as pre-processing. Support scale is a commonly known expression to account for the effective area the measurement should represent. A small interrogation area (IA) and time difference gives more details on particle displacement, but does not necessarily give more accurate results [17]. We performed a sensitivity study for different choices of IA and found that the minimum size of IA is defined by the number of moving particles within an area. The minimum scale was a grid length of the IA of $0.009 \mathrm{~m}$. An optimal IA size was obtained when the calculated mean displacement approached a constant value and further increasing IA dismissed the variability in the measured particle velocities (at $0.015 \mathrm{~m}$ ). For comparison, we manually traced particle motion of individual particles and calculated the mean velocity for increasing grid length. In Fig. 3 it is shown that the mean particle velocities based on manual particle tracking yielded a variability up to the maximum grid length we analysed $(0.036 \mathrm{~m})$. The corresponding standard deviation was $1.3 \mathrm{~m} / \mathrm{s}$. As we aim for our studies to measure spatial gradients in particle velocities over a dune slope, the minimum support scale depends on the number of particles in motion. For an average flow velocity of $0.5 \mathrm{~m} / \mathrm{s}$, this results in a minimum support scale of approximately $0.009 \mathrm{~m}$. Since we are interested in spatial gradients, the maximum support scale should also be below $0.036 \mathrm{~m}$, as with a longer grid length essential information is lost. The latter is illustrated in Fig. 4, showing that for increasing grid length, we lose information of spatial variability. At the scale of $0.026 \mathrm{~m}$ the observed particle velocity variability can be explained in the context of general onset and cessation of sediment transport, the effect of the reattachment zone and observed sweep/burst events. By decreasing the streamwise distance between cross-sections (as in Fig. 2), the variations in mean particle velocity induced by superimposed bed defects are distinguished as well. Summarising, automatic calculations with a support scale of $0.026 \mathrm{~m}$ allow for suitable quantification of streamwise velocity over a dune slope, while the signals from processes causing deviation from the mean such as flow structures and bed defects are pertained.

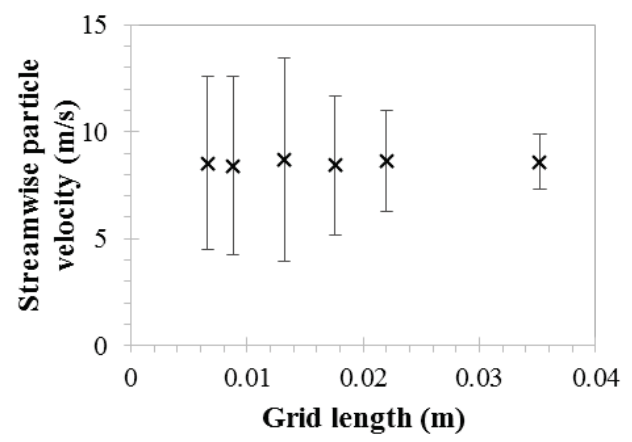

Fig. 3. The variability in the mean streamwise velocity decreases for increasing grid length up to the maximum grid length that we analyses of $0.036 \mathrm{~m}$. Variability is high for the manually traced particle velocities, while the mean of the velocities is constant for increasing grid lengths.

\section{Location of maximum transport over a dune crest}

The PIV-based measurements of mean particle velocity and measurements of mean particle activity from IoD of the bedload bridge the gap between individual particle motion studies and (non-local) sediment transport flux measurements. The measurements of the streamwise particle velocities over the dune slope showed an increase in mean streamwise 
particle velocity from the lower slope $(0.06 \mathrm{~m} / \mathrm{s})$ to the dune crest $(0.095 \mathrm{~m} / \mathrm{s})$ [21]. In Fig. $\mathbf{5}$, the mean streamwise particle velocity over the dune crest and part of the upper slope is presented ( $0-0.3 \mathrm{~m}$ upstream of the dune lee slope). The maximum streamwise particle velocity occurred $0.15 \mathrm{~m}$ upstream of the lee slope.

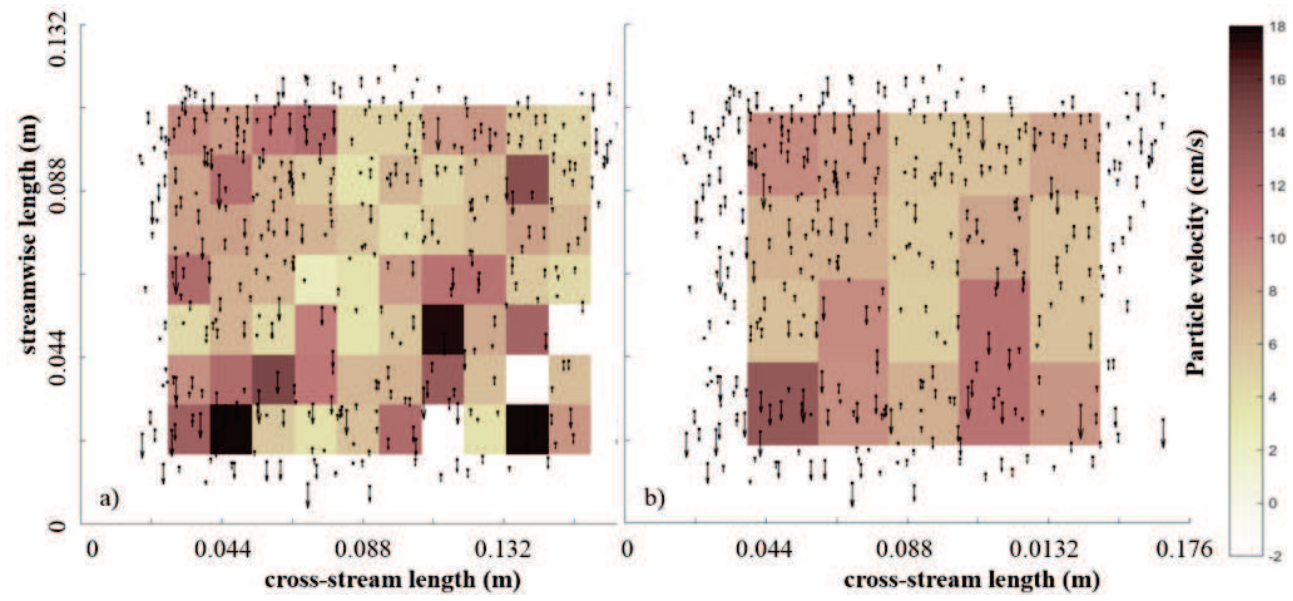

Fig. 4. Comparison of mean particle velocity based on manually tracking of individual particles for grid length of $0.013 \mathrm{~m}$ (a) and $0.022 \mathrm{~m}$ (b). Spatial variability in mean particle velocity decreases for increasing grid length.
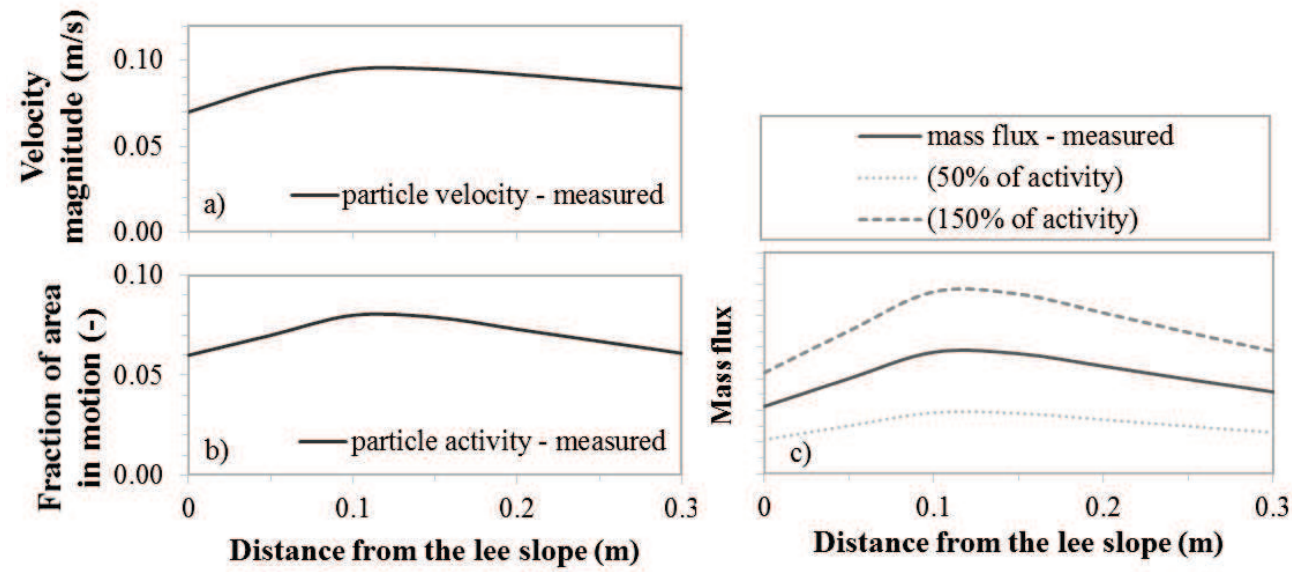

Fig. 5. Velocity magnitude from PIV-based method (a) and particle activity from IoD (b) over a dune slope. Subsequent measurements of mass flux (c) define the location of maximum transport over the dune crest. The location of maximum transport is $0.15 \mathrm{~m}$ upstream of the lee slope.

The particle activity was calculated from counting the number of particles and the pixels they covered in the IoD relative to the area of immobility. Particle activity increased from the lower slope to the dune crest. The maximum of the particle activity (area in motion) occurred at the same location as the maximum streamwise velocity. The maximum particle velocity and activity occurred at the same location and consequently the location of the maximum transport over the dune crest was identified $(0.15 \mathrm{~m}$ from the lee slope, black line in Fig. 6. To quantify the mass flux, derivation of particle volume and mass from the IoD is required. The validation of that method is not in the scope of this work. 


\section{Discussion}

Our measurements provide insight into the particle-scale and bedform-scale sediment transport. Maximum flux occurred $0.15 \mathrm{~m}$ upstream of the lee slope. The experimental dune field showed a regular spacing and was in a bedload dominated stage, while experimental run time implied an equilibrium state. The measurements imply that at the crest deposition occurred because maximum flux was located upstream and the dune was in a state of growth [4, 22]. Meaning, that the observed maximum flux was further upstream than expected based on the assumption of an equilibrium dune. One explanation is the occurrence of small superimposed bed forms near the location of maximum flux. Downstream of the superimposed bedform, a decrease in particle velocity and activity was observed, causing a negative deviation from the mean spatial gradient. A second explanation is that the method to calculate particle activity from IoD was influenced by particle velocity. We suggest, to shoot at a higher frequency, or with a smaller field of view, improve illumination of the scene, optimise the post-processing step of noise removal, and provide validation. Last, for interpretation and generalisation (of spatial gradients in bedload transport at the bed-form scale) simultaneous measurements of dune geometry, local flow and dune kinematics are essential.

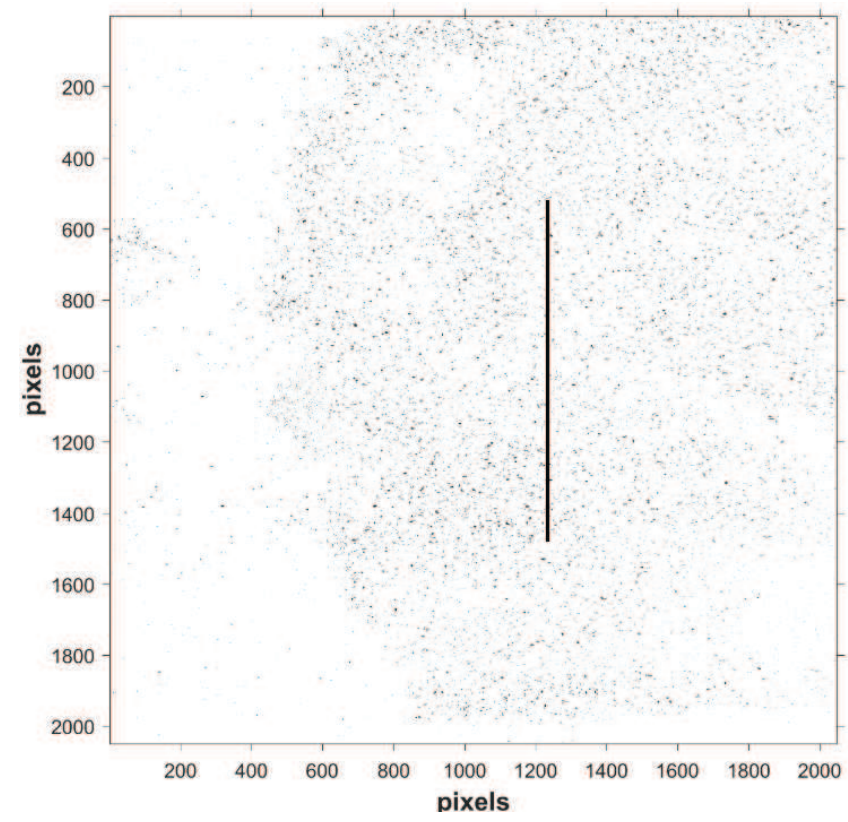

Fig. 6. IoD showing that few particles moved in the dune trough (left side) compared to the dune crest. Particle activity was highest at the location of the black line.

\section{Conclusions}

We introduce a PIV-based method based on images of difference (IoD) as an effective approach to obtain mean streamwise particle velocity, compared to individual particle tracking. Applying PIV for bedload particle motion allows for accurate quantification of streamwise velocity over a dune slope on a support scale of centimeters, while the signals from processes causing deviation from the mean, such as flow structures and bed defects, are pertained. The PIV-based method combined with IoD bridges the gap between 
individual particle motion studies and (non-local) sediment transport flux measurements and can be fully automated for the challenging condition of mobile bed and sand particles moving closely together. Findings are that mean streamwise particle velocity and particle activity increase over the dune slope. Those observations are aligned with our general knowledge of fluctuation of flow velocity over a dune caused by the dune geometry. We identify the location of maximum flux over the dune crest. Maximum flux is located further upstream of the dune crest than hypothesized. We conclude that for interpretation of our measurement of spatial gradients in bedload transport at the bed-form scale combined measurements of dune geometry, local flow and dune kinematics are essential. The present work will enable knowledge advances in sediment transport for superimposed bed forms and sweep/burst events.

\section{Acknowledgements}

Acknowledgements go to M.G. Kleinhans for reviews and discussions and to Geoff Kirby, Trevor Patrick, Wei Li and Richard Wilson for technical support.

\section{References}

1. J. Best, J Geophys Res-Earth, 110, (2005).

2. T. Maddux, J. Nelson, and S. McLean, J Geophys Res-Earth, 108, (2003).

3. S. R. McLean, Earth Sc. Revws, 29, (1990).

4. F. Engelund and J. Fredsøe, Ann. Rev. Fluid Mech., 14 (1982).

5. J. G. Venditti, C. Y. M. Lin, and M. Kazemi, Sedimentology, 63, (2016).

6. E. Lajeunesse, L. Malverti, and F. Charru, J Geophys Res-Earth, 115, (2010).

7. J. C. Roseberry, M. W. Schmeeckle, and D. J. Furbish, J Geophys Res-Earth, 117, (2012).

8. S. L. Fathel, D. J. Furbish, and M. W. Schmeeckle, J Geophys Res-Earth, 120, (2015).

9. E. M. Yager and M. W. Schmeeckle, J Geophys Res-Earth, 118, (2013).

10. A. Radice, S. Malavasi, and F. Ballio, Exp, in Fluids, 41, (2006).

11. J. Shim and J. G. Duan, Internat J. of Sediment Res., 32, (2017).

12. W. Brevis, Y. Nino, and G. H. Jirka, Exp Fluids, 50, (2011).

13. T. G. Drake, R. L. Shreve, W. E. Dietrich, P. J. Whiting, and L. B. Leopold, J. of Fluid Mech, 192, (1988).

14. Y. Nino and M. Garcia, J. Hydraul. Eng., 124, (1998).

15. J. Campagnol, A. Radice, R. Nokes, V. Bulankina, A. Lescova, and F. Ballio, Journal of Hydraulic Research, 51, (2013).

16. M. Tal, P. Frey, W. Kim, E. Lajeunesse, A. Limare, and F. Métivier, in Fluvial Remote Sensing for Science and Management, (2012).

17. Q. W. Lewis and B. L. Rhoads, Water Resources Res., 51, (2015).

18. E. Kwoll, J. G. Venditti, R. W. Bradley, and C. Winter, J Geophys Res-Earth, 122, (2017).

19. W. Thielicke and E. J. Stamhuis, J. of open res. software, 2, (2014).

20. K. G. Heays, H. Friedrich, B. W. Melville, and R. Nokes, J. Hydraul. Eng., 140, (2014).

21. R. C. Terwisscha van Scheltinga, H. Friedrich, and G. Coco, Measurements of sand particle velocities over a dune slope using high-frequency image capturing, (submitted)

22. M. Colombini, J. of Fluid Mech, 502, (2004). 